\title{
Hadronic Charm Decays from B Factories
}

\author{
H. R. Band ${ }^{a}$

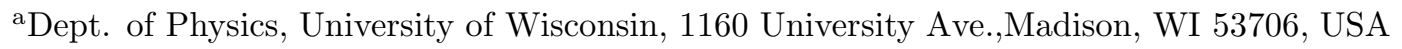

The B factories, KEKB and PEPII, provide enormous samples of charmed mesons and baryons as well as $B \bar{B}$ events. The BELLE and BaBar collaborations have discovered many new particles containing charm quarks in the last few years and have measured their properties with increasing precision. The current status and most recent studies of these charm particle properties is briefly reviewed.

\section{Introduction}

The B-Factory collaborations have produced many new charm meson and baryon analyses for the 2006 summer conferences featuring larger data sets, expanded particle searches and increased sophistication. Covering the many results from BELLE and BaBar is impossible for this short writeup. Only a sampling of the newest discoveries will be described with minimal details of the different analysis techniques. My apologies to the authors whose work has been omitted in the interest of brevity.

\section{1. $B$ and $C$ factories}

The PEPII and KEKB asymmetric B-Factories were built to supply large numbers of $B \bar{B}$ mesons for $\mathrm{CP}$ asymmetry measurements. However, they also produce copious amounts of charmed baryons and mesons in $c \bar{c}$ continuum production $(1.3 \mathrm{nb})$ or in B decays. Including on-peak $\Upsilon(4 \mathrm{~S})$ and offpeak data, BELLE and BaBar observe $30 \%$ more $c \bar{c}$ than $B \bar{B}$ events. Therefore the B-Factories should be also considered C-Factories.

Both PEPII and KEKB have exceeded their design luminosities and have integrated over 1 $\mathrm{ab}^{-1}$ between them $\left(630 \mathrm{fb}^{-1}\right.$ BELLE, $390 \mathrm{fb}^{-1}$ BaBar). However, most of the following analyses use only part of the available data sets. The results quoted here should be considered preliminary unless referenced by a journal publication. Use of charge-conjugate decays is always implied unless stated otherwise.

\section{Charmed mesons}

The first new charmed mesons observed by BaBar [1,4], CLEO [2], and BELLE [3] were the $D_{s 0}(2317)^{+}$and $D_{s 1}(2460)^{+}$. Their masses were lower than expected from heavy quark potential models $[5,6]$ which had successfully explained the masses of the previously detected $c \bar{s}$ mesons. Despite speculation about the possible exotic nature of these new states they were soon shown [7] to have the spin and parity $J^{P}=0^{+}, 1^{+}$expected for the next lowest mass $c \bar{s}$ mesons.

\section{1. $D_{s J}$ states}

The BaBar Collaboration has systematically studied $c \bar{c}$ continuum decays to final states containing $D_{s} \rightarrow K^{+} K^{-} \pi^{+}$in a data sample of 232 $\mathrm{fb}^{-1}[8]$. Backgrounds were reduced by requiring that either the $K^{+} K^{-}$originate in a $\phi$ decay or that the $K^{-} \pi^{+}$originate in a $K^{*}(892)$ decay. Tight cuts on the $D_{s}$ center of mass momentum and production angles further reduced backgrounds, yielding 400,000 $D_{s}$ candidates in a $0.25 \mathrm{GeV} / \mathrm{c}^{2}$ window centered on the nominal $D_{s}$ mass. Sidebands outside the signal region were used to estimate combinatorial backgrounds.

$D_{s J}$ mesons were searched for in the following decay modes: $D_{s} \gamma, D_{s} \pi^{0}, D_{s} \pi^{0} \gamma, D_{s} \pi^{0} \pi^{0}$, $D_{s} \gamma \gamma, D_{s} \pi^{+}, D_{s} \pi^{-}, D_{s} \pi^{+} \pi^{-}$. The invariant mass distributions were often complicated by reflections from other $D_{s J}$ states in which a $\gamma$ or $\pi^{0}$ were missed. In some cases the reflection was partially under the desired signal peak and required careful modeling to fit. No significant sig- 
Table 1

Cross section yields $\left(\sigma\left(D_{s J}\right) \times B\left(D_{s J} \rightarrow D_{s} X\right) \times B\left(D_{s}^{+} \rightarrow \phi \pi^{+}\right)\right.$or limits by decay mode. Cross sections or $95 \%$ CL limits are in $\mathrm{fb}^{-1}$.

\begin{tabular}{lllll}
\hline Final state & $D_{s} \gamma$ & $D_{s} \pi^{0}$ & $D_{s} \pi^{0} \gamma$ & $D_{s} \pi^{+} \pi^{-}$ \\
\hline$D_{s 0}(2317)^{+}$ & $\leq 15.7$ & $115.8 \pm 2.9 \pm 8.7$ & $\leq 16.7$ & $\leq 0.6$ \\
$D_{s 1}(2460)^{+}$ & $14.4 \pm 1.0 \pm 1.4$ & $\leq 1.7$ & $42.7 \pm 3.5 \pm 4.2$ & $3.3 \pm 0.5 \pm 0.6$ \\
$D_{s 1}(2536)^{+}$ & & & & $5.2 \pm 0.7 \pm 0.4$ \\
\hline
\end{tabular}

The experimental values are from ref.[8].

nals were seen in the $D_{s} \pi^{+}, D_{s} \pi^{-}$, and $D_{s} \gamma \gamma$ final states. The observed yields for the other final states are shown in Tab. 1. Combining the mass and width fits from the different final states the masses were measured as follows: $M\left(D_{s 0}(2317)^{+}\right)=2319.6 \pm 0.2 \pm 1.4 \mathrm{MeV} / \mathrm{c}^{2}, \Gamma \leq$ $3.8 \mathrm{MeV} / \mathrm{c}^{2} ; M\left(D_{s 1}(2460)^{+}\right)=2460.1 \pm 0.2 \pm$ $0.8 \mathrm{MeV} / \mathrm{c}^{2}, \Gamma \leq 3.5 \mathrm{MeV} / \mathrm{c}^{2} ; M\left(D_{s 1}(2536)^{+}\right)=$ $2534.6 \pm 0.2 \pm 0.7 \mathrm{MeV} / \mathrm{c}^{2}, \Gamma \leq 2.5 \mathrm{MeV} / \mathrm{c}^{2}$.

BaBar has looked for new $D_{s J}$ states [9] in the continuum decaying to $D^{0} K^{+}$or $D^{+} K_{s}$ using over $2 \times 10^{6}$ reconstructed D's. The DK invariant mass distribution is shown in Fig. 1. When the scale is expanded above the prominent $D_{s 2}(2573)^{+}$peak, two bumps are seen in all of the DK final states. After combining modes and subtracting the expected backgrounds(Fig. 1d), a mass peak attributed to a new $D_{s J}$ is observed at $M\left(D_{s J}(2860)^{+}\right)=2856.2 \pm 0.3 \pm 1.0 \mathrm{MeV} / \mathrm{c}^{2}, \Gamma=$ $47 \pm 7 \pm 10 \mathrm{MeV} / \mathrm{c}^{2}$. A second structure at $M(X)=2688 \pm 4 \pm 3 \mathrm{MeV} / \mathrm{c}^{2}, \Gamma=112 \pm$ $7 \pm 36 \mathrm{MeV} / \mathrm{c}^{2}$ has been tentatively labeled as $\mathrm{X}(2690)$ and is being studied further.

BELLE has looked at $B \rightarrow \overline{D^{0}} D^{0} K^{+}[10]$ decay modes. In addition to signals from the $\Psi(3770)$ and $\Psi(4160)$ they claim observation of a new $D_{s J}$ state at $M=2715 \pm 11 \pm 14 \mathrm{MeV} / \mathrm{c}^{2}, \Gamma=$ $115 \pm 20 \pm 36 \mathrm{MeV} / \mathrm{c}^{2}$. A spin analysis of the decays favors a $J^{P}=1^{-}$assignment. Since the $D_{s 1}(2700)$ and the $\mathrm{X}(2690)$ have very similar masses and widths it is possible that they reflect 2 different decay modes of the new state.

\subsection{D branching fractions}

A powerful technique used by BELLE and BaBar to measure the absolute branching fractions uses $B \bar{B}$ events in which one of the B's has been fully reconstructed. In a BaBar analysis [11] a $D^{(*)+,-, 0}$ or $D_{s}^{(*)-}$ is reconstructed $\left(D_{\text {meas }}\right)$ from the decay of the other B and is used to calculate the mass of the remaining particles, $D_{X}$ in the event. From two separate classes of events with $D_{\text {meas }}=D_{s}^{(*)+}$ and $D_{X}=D_{s}^{(*)-}$ BaBar has measured the branching fraction of $D_{s}^{-} \rightarrow \phi \pi^{-}$to be $\mathrm{BF}=4.62 \pm 0.36 \pm 0.51 \%$, consistent with, but more precise than the PDG [12] value of $4.3 \pm 1.2 \%$.

The recoil mass for one particular mode is plotted in Fig. 2 when a $D^{*+}$ is required. Clear signals for $D_{s 1}(2460)^{-}, D_{s}^{*-}$, and $D_{s}^{-}$are seen. When $D_{X}=D_{s 1}(2460)^{+}$, the data can then be combined with the previously measured exclusive branching fractions [13] such as $\mathrm{B}\left(B^{0} \rightarrow\right.$ $\left.D^{(*)} D_{s 1}(2460)^{-}\right) \times B\left(D_{s 1}(2460)^{-} \rightarrow D_{s}^{*-} \gamma\right)$ to give the following absolute branching fractions: $\mathrm{BF}\left(D_{s 1}(2460)^{+} \rightarrow D_{s}^{*+} \pi^{0}\right)=0.56 \pm 0.13 \pm 0.09$ and $\operatorname{BF}\left(D_{s 1}(2460)^{+} \rightarrow D_{s}^{*+} \gamma\right)=0.16 \pm 0.04 \pm$ 0.03 .

BELLE uses a similar technique in two-body final states containing $D_{s 1}(2536) D_{s}^{*}$ from continuum $c \bar{c}$ production to measure the branching fraction of the $D_{s}^{+}$to $K^{+} K^{-} \pi^{+}$[14]. Either the $D_{s 1}(2536)^{+}$(in the decay modes $D^{* 0} K^{+}$or $\left.D^{*+} K_{s}\right)$ or the $D_{s}^{*}\left(K^{+} K^{-} \pi^{+}\right)$is fully reconstructed and the recoil mass of the other particles in the event are calculated. The $D_{s}^{*}$ ( or $\left.D_{s 1}(2536)^{+}\right)$appears as a peak in the recoil mass distribution. To enhance the signal peak and to further suppress backgrounds the $D_{s}^{*}$ is required to decay to $D_{s}^{+} \gamma$. The $\gamma$ from the signal side is used to calculate a $\Delta M_{\text {recoil }}\left(D_{s 1} \gamma\right)$ mass distribution. BELLE finds $\mathrm{B}\left(D_{s}^{*} \rightarrow K^{+} K^{-} \pi^{+}\right)=$ $4.1 \pm 0.4 \pm 0.4 \%$, substantially reducing the error on the present $\mathrm{PDG}[12]$ value $(5.2 \pm 0.9 \%)$. 

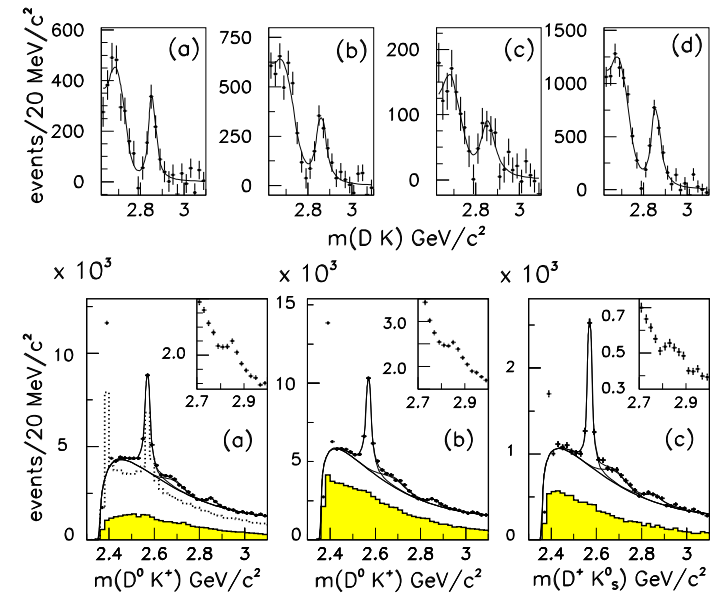

Figure 1. Bottom: $D K$ invariant mass for a) $D^{o} \rightarrow K^{-} \pi^{+}$, b) $D^{o} \rightarrow K^{-} \pi^{+} \pi^{o}$, and c) $D^{+} \rightarrow$ $K^{-} \pi^{+} \pi^{+}$. Top: background subtracted $D^{\circ} K^{+}$ invariant mass for a), b), c) as above, and d) sum of all 3 decay modes. The large peak in the top plots is from the $D_{s 2}(2573)^{+}$decay. The peak at $2860 \mathrm{GeV} / \mathrm{c}^{2}$ is fit by a Breit-Wigner and is attributed to a new state $D_{s j}(2860)^{+}$. A possible excitation is also seen at $2690 \mathrm{GeV} / \mathrm{c}^{2}$

Improved branching fraction measurements in rare $\mathrm{D}$ decays would benefit $D^{0} \overline{D^{0}}$ mixing analyses by helping to understand the size of $\mathrm{SU}(3)$ violating effects. The best strategy is to combine a cross section ratio measurement of the rare decay to a well measured reference decay. BaBar [15] has measured the relative production of $D^{+} \rightarrow h^{+} \pi^{0}$ ( where $\mathrm{h}$ is a $\mathrm{K}$ or $\pi$ ) to $D^{+} \rightarrow K^{-} \pi^{+} \pi^{+}$(ref). The $D^{+}$was required to originate from an $D^{*+} \rightarrow D^{+} \pi^{0}$ decay to reduce backgrounds. Using the branching fraction $B\left(D^{+} \rightarrow K^{-} \pi^{+} \pi^{+}\right)$from CLEO [16] of $9.4 \pm$ $0.3 \times 10^{-2}$, BaBar measures $\mathrm{B}\left(D^{+} \rightarrow \pi^{+} \pi^{0}\right)=$ $1.25 \pm 0.10 \pm 0.9 \pm 0.04($ ref $) \times 10^{-3}$ and $\mathrm{B}\left(D^{+} \rightarrow\right.$ $\left.K^{+} \pi^{0}\right)=2.52 \pm 0.47 \pm 0.25 \pm 0.08$ (ref) $\times 10^{-4}$. This is the first observation of the doubly-Cabbibo suppressed $K^{+} \pi^{0}$ mode.

The two-body Cabbibo suppressed decays of

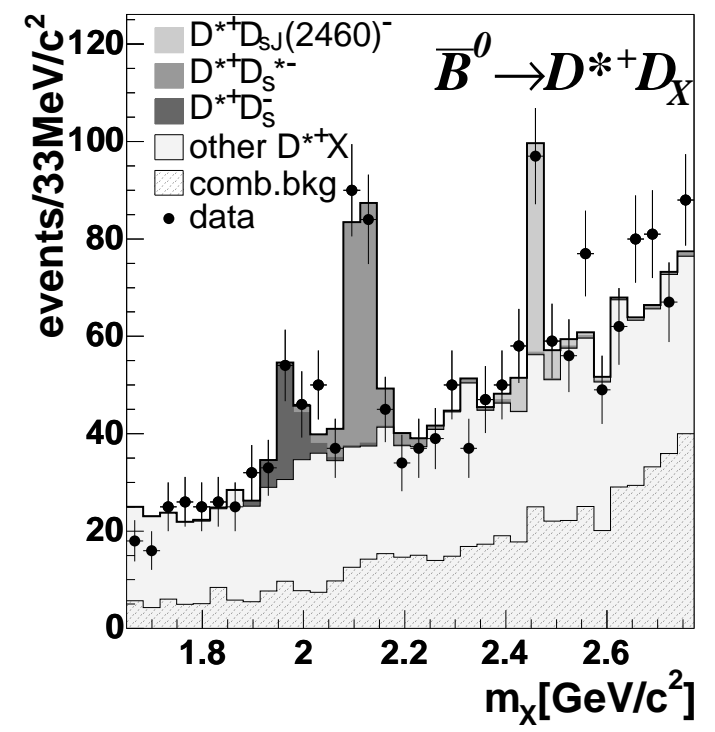

Figure 2. Missing mass of $\mathrm{B}$ decays to $D^{*+} \mathrm{X}$. The other B in the event has been fully reconstructed. Expected signal and background yields are shown by the shaded histograms.

the $D^{0}$ are well measured and show anomalous suppression of the $\pi^{+} \pi^{-}$mode versus the $K^{+} K^{-}$mode $\left(\pi^{+} \pi^{-}\right.$is favored by phase space). The three body modes may not be similarly suppressed but are not well measured. In a recent study [17] BaBar measures the branching ratio of $\pi^{+} \pi^{-} \pi^{0}$ or $K^{+} K^{-} \pi^{0}$ to the Cabbibo favored $K^{-} \pi^{+} \pi^{0}$ mode. BaBar finds the branching ratio of $\mathrm{B}\left(\pi^{+} \pi^{-} \pi^{0}\right) / \mathrm{B}\left(K^{-} \pi^{+} \pi^{0}\right)$ to be $10.59 \pm 0.06 \pm 0.13 \%$ (previous PDG result $8.4 \pm 3.11 \%)$. Belle [18] has also measured this mode to be $9.90 \pm 0.1 \pm 0.3 \%$. For $\mathrm{B}\left(K^{+} K^{-} \pi^{0}\right) /$ $\mathrm{B}\left(K^{-} \pi^{+} \pi^{0}\right) \mathrm{BaBar}$ finds $2.37 \pm 0.03 \pm 0.04 \%$ (previous PDG result $0.95 \pm 0.26 \%$ ). For these three body modes the branching fractions follow those expected from phase space. 


\section{Charmed baryons}

Prior to this year all of the $J^{P}=1 / 2^{+}$and most of the $J^{P}=3 / 2^{+}$baryons $(\mathrm{L}=0)$ containing a single charm quark had been observed. Several higher orbital exicitations $(\mathrm{L}=1)$ were also seen. Adding interest to this area, the SELEX collaboration reported observation of doubly charmed baryons in several decay modes.

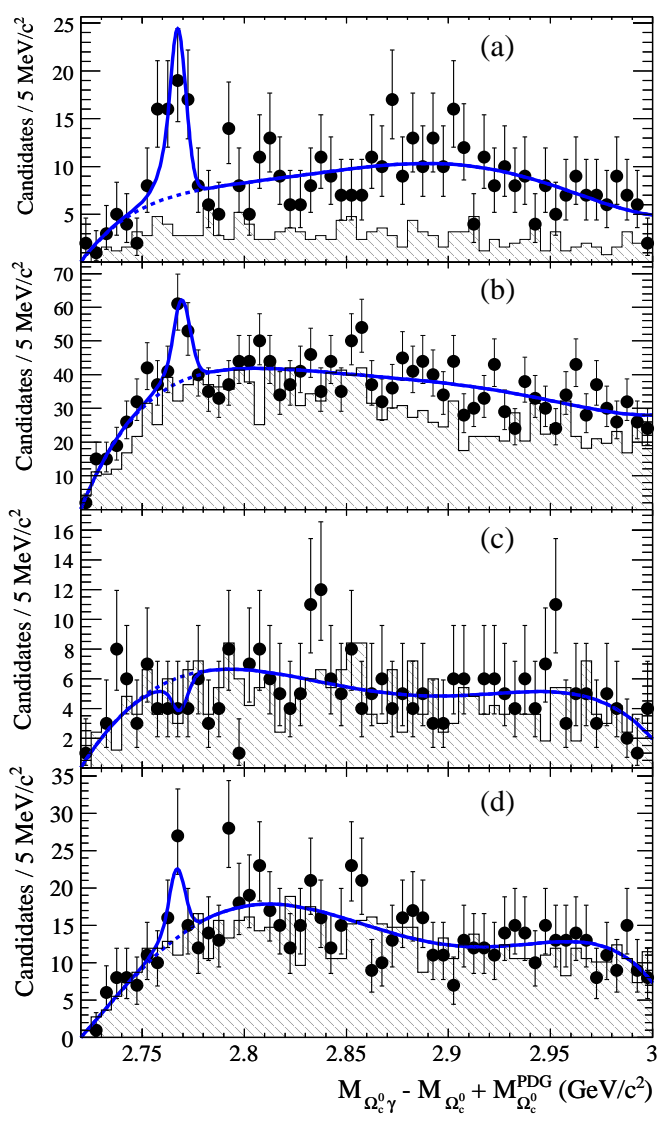

Figure 3. Invariant mass distribution of $\Omega_{c}^{0} \gamma$ candidates with $\Omega_{c}^{0} \rightarrow$ : (a) $\Omega^{-} \pi^{+}$, (b) $\Omega^{-} \pi^{+} \pi^{0}$, (c) $\Omega^{-} \pi^{+} \pi^{-} \pi^{+}$, and (d) $\Xi^{-} K^{-} \pi^{+} \pi^{-}$. The data are shown as solid points and the background estimated from the $\Omega_{c}$ sidebands is shaded.
3.1. $\Xi_{c}(2980)^{+}, \Xi_{c}(3077)^{+}$

Both BELLE [19] and BaBar [20] have searched for evidence of doubly-charmed baryons in the same final states as observed by SELEX without success. Belle did not observe the $\Xi_{c c}(3520)^{+}$reported by SELEX [21] in the $\Lambda_{c}^{+} K^{-} \pi^{+}$final state but did observe two new baryons [19] with mass and widths of $2978.5 \pm 2.1 \pm 2.0 \mathrm{MeV} / \mathrm{c}^{2}, \Gamma=$ $43.52 \pm 7.5 \pm 7.0 \mathrm{MeV} / \mathrm{c}^{2}$ and $3076.7 \pm 0.9 \pm$ $0.5 \mathrm{MeV} / \mathrm{c}^{2}, \Gamma=6.22 \pm 1.2 \pm 0.8 \mathrm{MeV} / \mathrm{c}^{2}$. The states were also seen in the isospin partner states confirming the assignment as $\Xi_{c}(2980)^{+}$and $\Xi_{c}(3077)^{+}$. These observations were confirmed by BaBar [22].

\section{2. $\Omega_{c}^{*}$}

The $\Omega_{c}^{*}(c s s)$ was the last unobserved $(\mathrm{L}=0)$ singly-charmed baryon. Lattice QCD calculations [23] predicted a mass difference between the $\Omega_{c}^{*}$ and $\Omega_{c}$ of $\Delta M=50-73 \mathrm{MeV} / \mathrm{c}^{2}$. BaBar reconstructed nearly $300 \Omega_{c}^{0}$ candidates in final states containing $\Omega^{-} \pi^{+}, \Omega^{-} \pi^{+} \pi^{0}, \Omega^{-} \pi^{+} \pi^{-} \pi^{+}$, and $\Xi^{-} K^{-} \pi^{+} \pi^{-}$. The $\Omega_{c}^{0}$ candidate was combined with a $\gamma(\mathrm{E} \geq 80 \mathrm{MeV})$ to make the invariant mass distribution of these final states shown in Fig. 3. Signal peaks from $\Omega_{c}^{*}$ are seen in three of the four channels. The measured mass difference [24] $\left(\gamma \Omega_{c}^{0}\right)-\mathrm{M}\left(\Omega_{c}^{0}\right)$ is determined to be $\Delta M=70.8 \pm 1.0 \pm 1.1 \mathrm{MeV} / \mathrm{c}^{2}$.

\section{3. $\Lambda_{c}(2940)^{+}$}

BaBar also searched for new baryon states in $D^{0} \mathrm{p}$ where the $D^{0}$ decays to either $K^{-} \pi^{+}$or $K^{-} \pi^{+} \pi^{+} \pi^{-}$. The $D^{0} \mathrm{p}$ invariant mass is shown in Fig. 4. The $\Lambda_{c}(2880)^{+}$previously observed in $\Lambda_{c} \pi^{+} \pi^{+}$by CLEO [25] is evident as well as a new $\Lambda_{c}$ state[26], with a mass and width of $2938.8 \pm$ $1.3 \pm 1.0 \mathrm{MeV} / \mathrm{c}^{2}, \Gamma=17.52 \pm 5.2 \pm 5.9 \mathrm{MeV} / \mathrm{c}^{2}$. No evidence for neutral or doubly-charged partners were observed supporting the assignment as $\Lambda_{c}(2940)^{+}$.

\section{Summary}

New charm meson and baryon states are still being discovered by the BELLE and BaBar collaborations. Since the B-Factories are expected to more than double their data sets in the next 


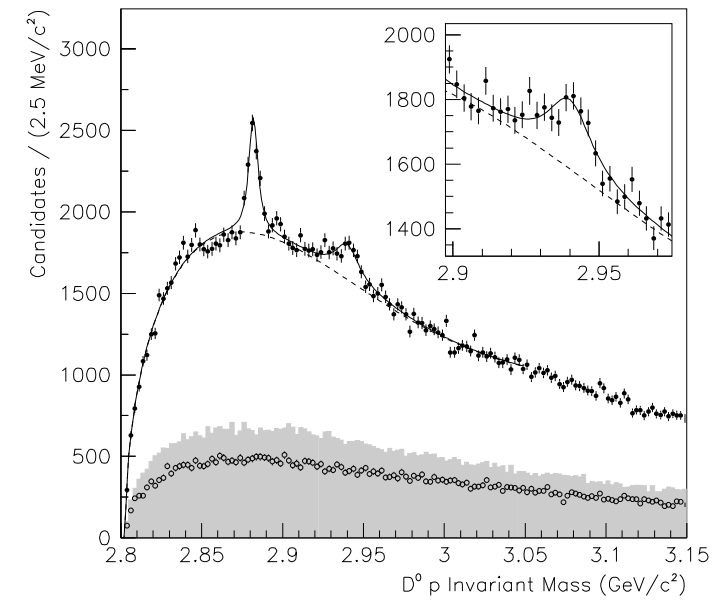

Figure 4. Reconstructed $D^{0} \mathrm{p}$ invariant $\operatorname{mass}\left(\mathrm{GeV} / \mathrm{c}^{2}\right)$ spectrum. The data (solid points), estimated background from the $D^{0}$ mass sidebands (histogram), and wrong sign $D^{0} \mathrm{p}$ (open circles) are plotted.

two years and since most of the analyses reported in this article use only a fraction of the presently available data, the statistical errors will be reduced by at least two. We can expect significantly improved branching fraction measurements and more observations of rare decay channels. We can also expect that the list of new charm states discovered by the B-Factory detectors will grow substantially.

\section{REFERENCES}

1. B. Aubert et al., BaBar Collaboration, Phys. Rev. Lett. 90 (2003) 242001.

2. D. Besson et al., CLEO Collaboration, Phys. Rev. D89 (2003) 032002.

3. K. Abe et al., BELLE Collaboration, Phys. Rev. Lett. 92 (2004) 012002.

4. B . Aubert et al., BaBar Collaboration, Phys. Rev. D69 (2004) 031101.

5. S. Godfrey and N. Isgur, Phys. Rev. D32 (1985) 189.
6. M. DiPiero and E. Eichten, Phys. Rev. D64 (2001) 114004.

7. K. Abe et al., BELLE Collaboration, Belle Conf-0461 (2004).

8. B. Aubert et al., BaBar Collaboration, arXiV:hep-ex/0605036.

9. B. Aubert et al., BaBar Collaboration, arXiV:hep-ex/0607082.

10. K. Abe et al., BELLE Collaboration, Belle Conf-0643(2006).

11. B. Aubert et al., BaBar Collaboration, arXiV:hep-ex/0605036.

12. W. M. Yao et al., Particle Data Group, J. Phys. G33 (2006) 1.

13. B. Aubert et al., BaBar Collaboration, Phys. Rev. Lett. 93 (2004) 181801.

14. K. Abe et al., BELLE Collaboration, Belle Conf-0612(2006).

15. B. Aubert et al., BaBar Collaboration, arXiV:hep-ex/0605044.

16. Q. Ye et al., CLEO Collaboration, Phys. Rev. Lett. 95 (2005) 121801.

17. B. Aubert et al., BaBar Collaboration, arXiV:hep-ex/0608009.

18. K. Abe et al., BELLE Collaboration, Belle Conf-0608(2006).

19. R. Chistor et al., BELLE Collaboration, arXiV:hep-ex/0606051.

20. B. Aubert et al., BaBar Collaboration, Phys. Rev D74 (2006) 011103.

21. M. Mattson et al, SELEX Collaboration, Phys. Rev. Lett. 89 (2002) 112001.

22. B. Aubert et al., BaBar Collaboration, arXiV:hep-ex/0607042.

23. N. Mathur et al., Phys. Rev. D66 (2002) 014512.

24. B. Aubert et al., BaBar Collaboration, arXiV:hep-ex/0608055.

25. M. Artuso et al., CLEO Collaboration, Phys. Rev. Lett. 86 (2001) 4470.

26. B. Aubert et al., BaBar Collaboration, arXiV:hep-ex/0603052. 